\title{
NMR-Based Metabolomics in Metal-Based Drug Research
}

\author{
Federica De Castro ${ }^{1}\left(\mathbb{D}\right.$, Michele Benedetti ${ }^{1,2, *} \mathbb{B}$, Laura Del Coco ${ }^{1}(\mathbb{D}$ and \\ Francesco Paolo Fanizzi 1,2,*(D)
}

1 Dipartimento di Scienze e Tecnologie Biologiche ed Ambientali, Università del Salento, Via Monteroni, I-73100 Lecce, Italy; federica.decastro@unisalento.it (F.D.C.); laura.delcoco@unisalento.it (L.D.C.)

2 Consorzio Interuniversitario di Ricerca in Chimica dei Metalli nei Sistemi Biologici (CIRCMSB), via Celso Ulpiani, 27, 70126 Bari, Italy

* Correspondence: michele.benedetti@unisalento.it (M.B.); fp.fanizzi@unisalento.it (F.P.F); Tel.: +39-0832-299264 (M.B.); +39-0832-299265 (F.P.F.); Fax: +39-0832-298626 (M.B. \& F.P.F.)

Received: 16 May 2019; Accepted: 13 June 2019; Published: 15 June 2019

\begin{abstract}
Thanks to recent advances in analytical technologies and statistical capabilities, the application field of metabolomics has increased significantly. Currently, this approach is used to investigate biological substrates looking for metabolic profile alterations, diseases markers, and drug effects. In particular, NMR spectroscopy has shown great potential as a detection technique, mainly for the ability to detect multiple (10s to 100s) metabolites at once without separation. Only in recent years has the NMR-based metabolomic approach been extended to investigate the cell metabolic alterations induced by metal-based antitumor drug administration. As expected, these studies are mainly focused on platinum complexes, but some palladium and ruthenium compounds are also under investigation. The use of a metabolomics approach was very effective in assessing tumor response to drugs and providing insights into the mechanism of action and resistance. Therefore, metabolomics may open new perspectives into the development of metal-based drugs. In particular, it has been shown that NMR-based, in vitro metabolomics is a powerful tool for detecting variations of the cell metabolites induced by the metal drug exposure, thus offering also the possibility of identifying specific markers for in vivo monitoring of tumor responsiveness to anticancer treatments.
\end{abstract}

Keywords: metabolomic; ${ }^{1} \mathrm{H}$ NMR Spectroscopy; antitumour drugs; metal drugs; platinum drugs

\section{Introduction}

In international scientific researches, the term "Omic" started to be used in the 1990s, when the Human Genome Project, whose aim was to determine the DNA nucleobases' sequences, was launched, in order to identify and map genes of the human genome (the "genomics" research field) [1]. The growth of scientific knowledge and the development of new technologies increased the need to further delimit and define the fields of related research, mainly focusing on the detection of mRNA (transcriptomics), proteins (proteomics), and metabolites (metabolomics) in specific biological samples [2].

These research strategies resulted in many applications and still have much potential. In fact, Omics technologies can be applied, not only for the understanding of biological systems in a normal physiological state, but also to get insight for specific disease conditions. Indeed, these technologies can play a determinant role in screening, diagnosis, and prognosis, as well as in the understanding of diseases etiology [2].

"Metabolomics" is the latest Omics technology. Like the other Omics sciences (such as genomics, transcriptomics, and proteomics), metabolomics uses analytical technologies that allow the production of large amounts of information (big data). In the specific case of metabolomics, the analytical 
techniques are normally used to identify, characterize, and quantify small biological molecules that are involved in the structure, function, and dynamics of cells, tissues, or organisms [3]. In this respect, different from the others Omics sciences, which can only describe what might happen to a biological system in the future, metabolomics gives information about what happened in the considered system and producing the observed metabolites, thereby getting direct insight about the physiological status of the studied organism [4].

The importance of metabolic profiling of bio-fluids was recognized, for the first time, in the late 1990s with the introduction of the term "metabonomic". This was last used to describe the quantitative measure of the metabolic, multi-parametric, and time-correlated response that a living system gives to a (patho) physiological stimuli or to genetic modifications [5]. Instead, the later definition of "metabolomics" consists of the identification and quantification of all the main low-molecular-weight metabolites/intermediates that vary according to the physiological or pathological state of the cell, tissue, organ, or organism of a biological system [6-9]. However, although some confusion has crept into the field, the difference in the use of metabolomics and metabonomics over the recent years has been clearly described [10], and nowadays the two terms are often used interchangeably [11].

Metabolomic science was born with the important aims of (among others) population profiling (for identifying metabolome-wide associations and novel risk biomarkers), food material profiling (for identify origin and for a quality control), and individual profiling (for a personalized health care). Nevertheless, it is also currently used for studying the mechanism of drug toxicity/efficiency and to develop new drugs [12]. On the other hand, there are some limitations related to the use of the technique, mainly due to biological variance and environmental influence, the difficulty of measuring minor (low-concentration) metabolites and the complexity of the resulting data sets. Despite these limitations, there are many advantages in using a metabolomic approach. First of all, there is the lack of need for analyte pre-selection, but also the achievable robustness and stability of analytical platforms [10].

It is important to consider that metabolomics generally consist of the use of separation (Gas Chromatography, GC, Capillary Electrophoresis, CE, High Performance Liquid Chromatography, HPLC, Ultra High Performance Liquid Cromatography, UPLC) and detection techniques (Nuclear Magnetic Resonance, NMR, and Mass Spectrometry, MS). Traditional methods require separation and optimization of the separation condition each time, followed by identification. Often, multiple slow separations (up to $72 \mathrm{~h}$ per sample) are necessary, and intensive manual work is needed, in addition to constant supervision and high-level skills [7,10]. In this context, the use of NMR spectroscopy in metabolomics studies has been given high throughput, thanks to the potential of this specific technique. Firstly, there is the advantage of measuring multiple (10 to 100) metabolites at once, with no need of their physical separation and a very limited sample working up. Secondly, this technique is non-destructive, and can be quantitative (different from MS) but capable at the same time, to allow metabolic profiles or "fingerprint" collection of the examined biological samples.

Due to the recent strong improvements of NMR technique and technologies, the NMR-based metabolomic is becoming a fast-growing and powerful technology [7]. Such improvements are essentially related to more reliable spectrometers, especially highly-sensitive NMR probes, with versatile acquisition sequences that can allow faster performance and substantial NMR experiments. Both the development of powerful databases of metabolic data and efficient multivariate statistical methods have simplified the high complexity (number of spectra, number of groups, number of condition) of big data set handling. Nowadays, all these aspects have made NMR-based metabolic profiling an unbiased tool that can provide fully quantitative data for most of the components in a complex mixture [7].

\section{NMR Metabolomics in Cancer}

One of the biggest areas of metabolomic research has been the discovery of metabolic biomarkers and biopatterns testifying to alterations due to cancer [4]. Cancer is, in fact, a fatal malignancy 
worldwide, as reported in the results of the Global Cancer Statistics of the International Agency for Cancer Research (IARC). In 2018, 18.1 million new cancer cases were estimated worldwide, as well as about 10 million deaths, over a half of cancer patients. These estimated data fully justify the close attention of researchers for improved technologies, allowing not only studies on cancer progression, but also the discovery of new biomarkers for a rapid and early diagnosis [13].

At this regard, it was demonstrated that a hallmark of cancer is represented by a reprogrammed metabolism. This was reported for the first time in the 1920s, thanks to the pivotal research of Otto Warburg showing that the metabolic patterns of cancer cells are generally different from those of normal cells [14]. In brief, normal cells use mitochondria to oxidise glucose and convert glucose into lactate only under hypoxia conditions (Figure 1a). In contrast, cancer cells avidly consume glucose, even in the presence of oxygen, and this is strictly associated with a lactate production increase (and then excretion; Figure 1b), together with the consequent acidic pH of cancer cells [14-16]. This discovery, at that time defined as "the root cause of cancer", and subsequently simply indicated as "the Warburg effect", won him the Nobel Prize for Medicine [15,16]. By increasing knowledge and scientific research, it was therefore proved that the Warburg effect cannot really be considered "the root cause of cancer", today known to be determined by a combination of genetic and environmental triggering factors [17]. Nevertheless, the Warburg effect clearly describes how cellular respiration enzymes act in tumour cells, thereby opening new perspectives in the study of cancer [18]. In addition to the better understanding of cancer cells' physiology, some studies are specifically designed to "reverse the Warburg effect" [19]. This last approach is also believed to be a valid method for pharmacologically acting against cancer. Indeed, several works have targeted the increased glycolysis, with the aim of inhibiting lactate production and excretion [19-23], and have found some success in preclinical models [24-26].

\section{Normal cells}

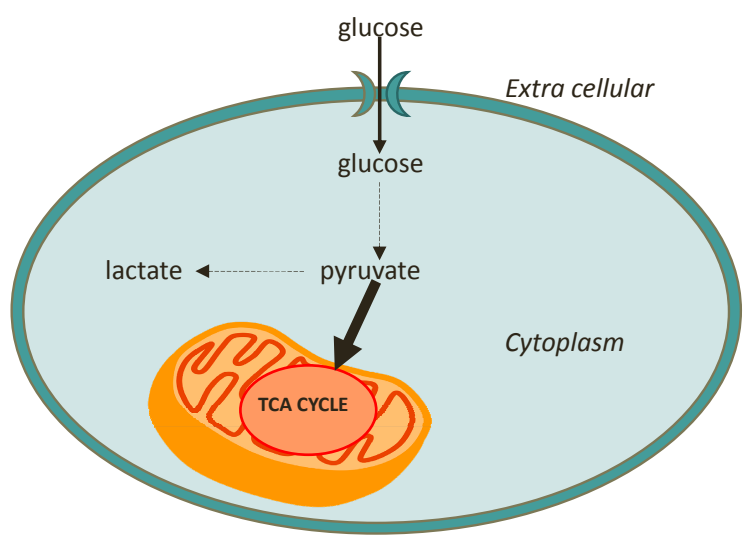

(a)

\section{Cancer cells (Warburg Effect)}

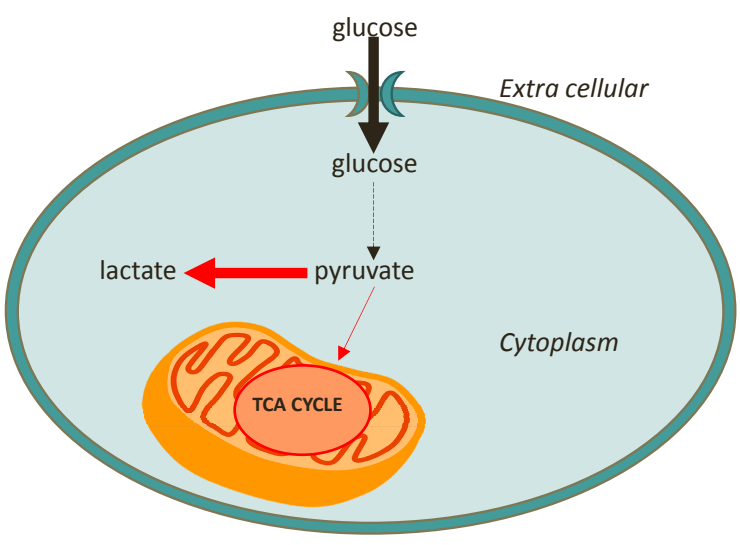

(b)

Figure 1. Schematic representation of (a) healthy cell metabolism in comparison to (b) cancer cell metabolism (the Warburg effect).

The Warburg effect has been confirmed in a variety of tumor types, including colorectal, breast, ovarian, lung, and glioblastoma cancers [14,18,27-32]. Furthermore, many studies have demonstrated that cancer cells are also dependent on fatty acid synthesis and glutaminolysis for proliferation [33]. All these differences with respect to normal cells suggest that targeting metabolic dependence could be a selective approach to treat cancer patients [27].

In this context, NMR spectroscopy, including in vivo magnetic resonance spectroscopy (MRS) and high-resolution magic angle spinning (HR-MAS NMR) analysis of tissue extracts, has been widely 
used to distinguish between different cell lines and tumor types [34]. As nuclear magnetic resonance spectroscopy is a high-throughput technology, it can be used to profile systemic metabolism in tumor diagnosis and prognosis [34]. NMR-based metabolomics have also been used to identify biomarkers for cancer, including hepatocellular carcinoma (HCC), colon cancer, ovarian cancer, breast cancer, prostate cancer, colorectal cancer, and others [4,35-41]. The aims of the studies have been to shorten the time to diagnosis, especially in cancers for which early detection and screening are difficult but would significantly affect therapeutic treatment decisions, as well as prognosis.

NMR metabolomics studies of cancer are applied to different kind of samples, such as bio-fluids (e.g., urine, whole blood, serum, plasma) and tissue/cell extracts (e.g., liver, brain, or kidney). Samples are normally examined directly, without extensive sample preparation, so that beside in vivo and in situ, a huge number of bio-fluids information are easily obtained [4,42-51].

The basic workflow of an NMR-based metabolomic approach starts with experimental design, including the evaluation of appropriate sample numbers to yield informative and statistically significant results, as well as biological and analytical experiments, such as sample preparation and NMR data acquisition. After this, the data preprocessing phase and subsequent data analysis follow. Then the metabolomics study requires the interpretation of the results using a multivariate data analysis, which usually consists of unsupervised and supervised methods (Figure 2).

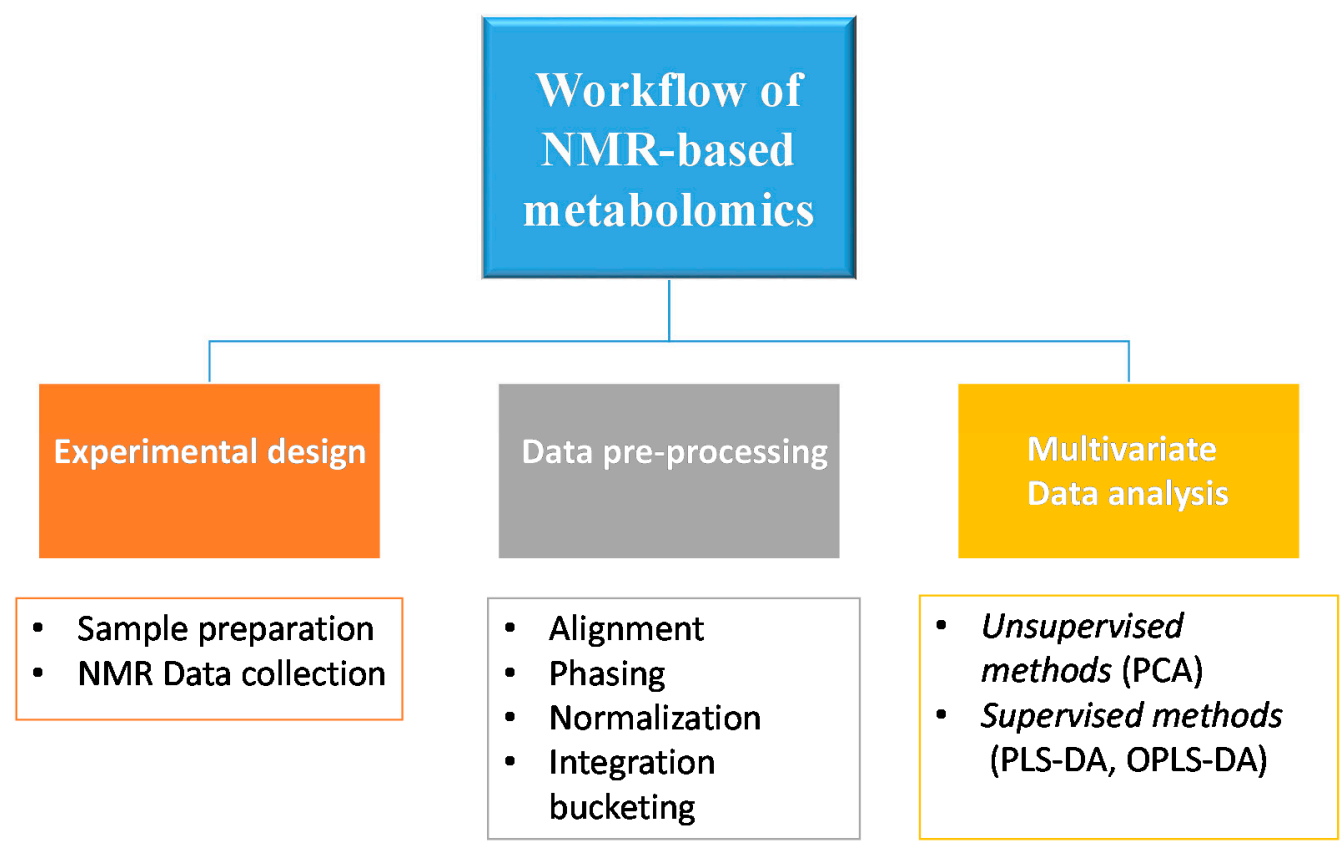

Figure 2. Schematic representation of the basic workflow of NMR-based metabolomics.

\section{Multivariate Data Analysis}

High-resolution NMR spectroscopy allows the detection of a wide range of chemical species simultaneously in a single experiment, by obtaining a large amount of data. For this reason, these spectroscopic techniques are generally coupled to a multivariate statistical treatment. Among available statistical methods, the most used in the NMR-based metabolomic studies were unsupervised principal component analysis (PCA), supervised partial-least-squares discriminant analysis (PLS-DA), and orthogonal partial-least-squares discriminant analysis (OPLS-DA).

Principal components analysis (PCA) has been widely used in several metabolomic studies, as it simply describes the intrinsic variation in the spectral data using a smaller number of factors or principal components (PCs). Each PC is a linear combination of the original data (the NMR variables), explaining the maximum amount of variance, not accounted by the previous PCs. Conversion of the spectral data to PCs results in two matrices, known as scores (corresponding to samples) and loadings 
(corresponding to NMR variables) [52]. By this unsupervised approach, it is possible to describe the general trend of samples and their potential clustering, depending on specific loadings (spectral variables) that may cause any cluster separation. In addition, there are other many unsupervised methods (e.g., $k$-means and hierarchical cluster analysis) also used to study how the data is distributed and to define groups within the samples. These methods essentially work on data forming a tree diagram, or dendrogram, which shows the relationships between samples. In general, the unsupervised analytical methods were used with the aim of identifying patterns in the data, as well as to obtain a general overview of the multivariate profiles. The further-supervised multivariate data analytical tools, such as projection to latent structure (PLS) and orthogonal projection to latent structure (OPLS), are tightly focused on the effects of interest, using any sample of a priori knowledge (e.g., class membership). Specifically, these methods relate the data matrix containing independent variables, such as spectral intensity values (defined as the $\mathrm{X}$ matrix), to a matrix containing dependent variables (e.g., measurements of response, such as toxicity or drug treatment) for those samples (Y matrix) $[52,53]$. This last ( $\mathrm{Y}$ matrix) can be defined by continuous ( $\mathrm{pH}$ values or the time course of the progression of a pathological effect), or discrete variables (group identity, health condition). In this case, a PLS discriminant analysis (PLS-DA), or its recent modification (OPLS-DA), is performed to examine the intrinsic variation in the data, as well as for classification purposes. In particular, the use of OPLS-DA consists in the separation of the $X$ data matrix (containing independent variables, such as spectral intensities) into two parts, one that is linearly related to $\mathrm{Y}$ and one that is unrelated (orthogonal) to $\mathrm{Y}$. This partitioning of the $X$ data facilitates model interpretation and its potential use in the prediction of new samples. The statistical model performance (robustness and predictive capability) is evaluated through permutation tests and cross-validation, together with the $\mathrm{R}^{2}$ and $\mathrm{Q}^{2}$ indices. The last two parameters $\left(R^{2}\right.$ and $\left.Q^{2}\right)$ respectively describe the total variations in the data and the predictability of models [53-56].

\section{The Potential of an NMR Metabolomics Approach in Monitoring the Response to Metal-Based Antitumor Drugs}

The development of a new drug is a fundamental step in medicine. It consists in identifying a general therapeutic area of interest, a specific disease to treat, and in most cases, the biological "target" [57]. In the past, the lack of complete knowledge of the action mechanism for a specific drug often resulted in the failure of clinical trials, as in the well-known case of Dimebon, a drug studied for Alzheimer's [58]. In fact, the Food and Drug Administration (FDA), the U.S. government agency responsible for regulating food and pharmaceutical use, does not require any specific understanding about the drugs' mechanism of action before starting clinical trials. Nevertheless, the last aspect is an essential step in the development of a new drug, because a clear understanding of the altered metabolic pathways due to a pharmacological treatment, as well as the action mechanism of a drug before it enters clinical trials, may prevent a late-stage failure [57]. A good strategy to detect treatment-related altered metabolic pathways is the monitoring of changes in the metabolome following the starting of chemotherapeutic treatment [59]. Indeed, the response to chemotherapy is often known to induce several metabolic alterations with respect to the physiological condition [59].

One of the most widely used anticancer drugs is cisplatin, a metal-based drug that acts by binding to genomic DNA targets. The discovery of cisplatin, a Pt(II) compound, by Barnett Rosenberg in 1960 represents a milestone in the history of metal-based compounds in the treatment of cancer [60]. Currently, cisplatin is used for the clinical management of patients affected by testicular, ovarian, head and neck, colorectal, bladder, and lung cancers.

Recently, many more metal-based compounds have been synthesized, either by redesigning the cisplatin chemical structure through ligand substitution, or by building completely new metal-based compounds. Therefore, regarding to pre-established structure-activity relationships (SARs), many unconventional $\mathrm{Pt}(\mathrm{II})$ and $\mathrm{Pt}(\mathrm{IV})$ compounds have been tested [61]. Nevertheless, other anticancer metal complexes-for example, complexes containing ruthenium ( $R u(I I)$ and $R u(I I I))[62,63])$, titanium 
(Ti(IV)), and gold ( $\mathrm{Au}(\mathrm{I})$ and $\mathrm{Au}(\mathrm{III})$ ) —have been studied [64]. In particular, Palladium (Pd) compounds have drawn particular attention, due to its similarity to $\mathrm{Pt}$ (II) (electronic structure and coordination chemistry) in showing favourable cytotoxic activity, despite both of their high lability [65-67]. Involved studies have essentially developed new metal-based drugs with the aim of enhanced safety and cytotoxic profiles, but also to overcome the resistance phenomena observed for cisplatin [68].

The use of NMR-based metabolomic approaches in the study of metal-based anticancer drugs is very new. Indeed, few papers, and only in recent years, have dealt with the use of this tool by studying the response of biological systems (biofluids, cells, and tissue) to metal-based drugs treatment in vitro and in vivo. This work covers the state-of-the-art survey of the NMR-based metabolomics approach adopted in metal-based drugs research, focusing on the possible applications and advantages of this investigation approach.

\subsection{NMR Metabolomics Studies of FDA-Approved Metal Complexes}

As expected, cisplatin is the first metal-drug studied by using an NMR metabolomic approach (see Figure 3a). Different samples, such as biofluids (urine, serum), and different cancer cell lines were examined in order to investigate cisplatin-induced metabolic alteration under different conditions of treatment, and in different tumour types. In particular, one of the interest topics of these scientific studies has been cisplatin-induced side effects.

At this regard, Portilla et al. have applied NMR-based metabolomic technology to the study of cisplatin-induced nephrotoxicity (one of the cisplatin-induced side effects). The research was performed through the analysis of urine samples of mice treated with cisplatin. Following administration of cisplatin for three days, the samples were collected and analysed by means of ${ }^{1} \mathrm{H}$ NMR spectroscopy coupled to multivariate data analysis (MVA). This study demonstrated that the presence of glucose, amino acids, and Kreb's cycle metabolites in the urine after cisplatin administration indicates the development of renal failure. The last specific cisplatin-induced metabolic NMR profile of urine in mice developing acute renal failure suggests that an injury-induced metabolic profile could be a biomarker of cisplatin-induced nephrotoxicity [69].

In further studies, Wen et al. extended the work of Portilla et al. to a larger number of animals, using a statistical approach by taking into consideration the intra-group variation (experiments are performed on the urine of 22 male Sprague-Dawley rats treated with cisplatin). Using NMR-based metabolomics combined with orthogonal partial least squares-discriminant analysis (OPLS-DA), the authors identified glucose, glycine, taurine, and branched amino acids as urinary biomarkers strictly associated with the renal toxicity induced by cisplatin [70].

As other known platinum-based drugs (such as carboplatin (Figure 3b) and oxaliplatin (Figure 3c)) approved by the FDA are largely used in the treatment of different tumours. In particular, oxaliplatin is used for the treatment of advanced colorectal cancer, though it can cause painful peripheral neuropathies, which pathophysiology has not been fully clarified. For this reason, Ferrier et al. used NMR metabolomics to monitor and investigate the effects of a polyamine-deficient diet in an animal model of oxaliplatin-induced acute pain hypersensitivity. The authors compared samples extracted from rat spinal dorsal horn, from rats fed with a normal diet, oxaliplatin-treated rats fed with a normal diet, and oxaliplatin-treated rats fed with a polyamine-deficient diet. Interestingly, they observed an increase of glutamate concentration in the spinal dorsal horn of rats after oxaliplatin treatment, as well as the ability of a polyamine-deficient diet to prevent neurotoxicity. This type of study is of crucial importance in oncology, especially taking in consideration that this type of injury (neurotoxicity) is often the cause of dose reduction or of discontinued treatment in cancer patients [71].

NMR-based metabolomics has also proved to be a useful tool in the prediction of treatment response to chemotherapy. The last is the principal aim of Jiang et al.'s research [72]. In this case, the experiments were focused on finding metabolites whose basal levels can predict the treatment response of 29 metastatic breast cancer (MBC) patients to gemcitabine-carboplatin chemotherapy. To make this, the ${ }^{1} \mathrm{H}$ NMR spectra of serum samples from patients with varying responses to subsequent 
chemotherapy were proved to be essential. Significantly, lower baseline levels of serum formate and acetate were observed in breast cancer patients who progress (MBC), with respect to those who achieve clinical benefits after receiving gemcitabine-carboplatin (GC) chemotherapy. These results indicated that formate and acetate might be used to select patients who should not be treated with GC chemotherapy, to avoid unnecessary adverse side effects.

Another NMR metabolomic application concerned with the possibility of distinguishing patients who are sensitive or insensitive to chemotherapy. In the interesting research of $\mathrm{Xu}$ et al., the serum of patients undergoing a platinum-based combined chemotherapy for lung cancer was analysed. The work demonstrated that insensitive subjects can be identified from the sensitive ones by observed up-regulation of glucose and taurine, but reduced alanine and lactate concentrations in the serum. The serum metabolic profile of patients can be therefore a valuable tool of their response to platinum-based combination chemotherapy [73].

In addition to the analysis of bio-fluids with NMR-metabolomics, the analysis of tumour cells gave high throughput regarding a drug action mechanism or for peculiar metabolites expressed in a specific type of tumour. This could be defined as the first step toward understanding drug synergism and a definition of new drug efficacy biomarkers. Duarte et al. deeply investigated the cisplatin treatment response in human lung cells and MG-63 osteosarcoma cells (in the last cell line, considering the comparison with doxorubicin and methotrexate as well) [74,75]. Their research strongly supports the ability of NMR metabolomics to measure cellular responses to different drug treatments, confirmed by the found potential biomarkers of cisplatin treatment response in the considered tumour cell lines.

Moreover, the dose-responsive effects of a metal-based drug could be also defined by NMR metabolomics. An example is the investigation of cisplatin response in human liver (L02) cells, described by Liu et al. [76]. Through the OPLS-DA analysis of data obtained from ${ }^{1} \mathrm{H}$ NMR spectra of L02 cell extracts (from control and cisplatin-treated groups), a clear discrimination was found. The study strongly supports the advantage of a global analysis using metabolomics, in order get an insightful view into the dose-response relationship, rather than using a single endpoint at the molecular level.

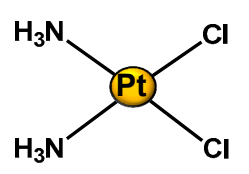

(a)

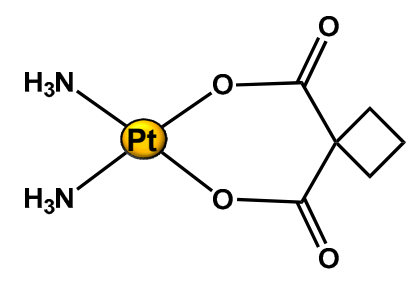

(b)

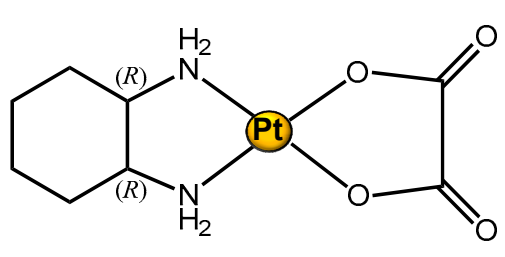

(c)

Figure 3. Structure of $\mathrm{Pt}(\mathrm{II})$ complexes studied through an ${ }^{1} \mathrm{H}$ NMR metabolomic approach. (a) Cisplatin; (b) Carboplatin; and (c) Oxaliplatin.

\subsection{NMR Metabolomic Studies of New Metal Complexes}

Through the NMR-metabolomics investigation of the effects of approved and widely used metal drugs (such as cisplatin, carboplatin, and oxaliplatin), this new field helps to discover how a new metal drug acts in a biological system, and how to define the specific target of the drug. An example is the study of Vermathen et al. on a new ruthenium complex. They used this technique to profile cells in response to treatment with a hexacationic ruthenium metallaprism (Figure 4a) in three different cell lines: A2780 (human ovarian cancer cells), A2780cisR (cisplatin resistant cells), and HEK-293 (human embryonic kidney cells). This research revealed a different response depending both on the cell type and incubation time. The observed different metabolic profile is mainly due to changes in level expression of lipids, choline-containing compounds, glutamate and glutathione, nucleotide sugars, lactate, and some amino acids. The time-dependent metabolic response patterns suggest that A2780 
cells on the one hand, and HEK-293 and A2780cisR cells on the other, may follow different cell-death pathways and exist in different temporal stages thereof [77].

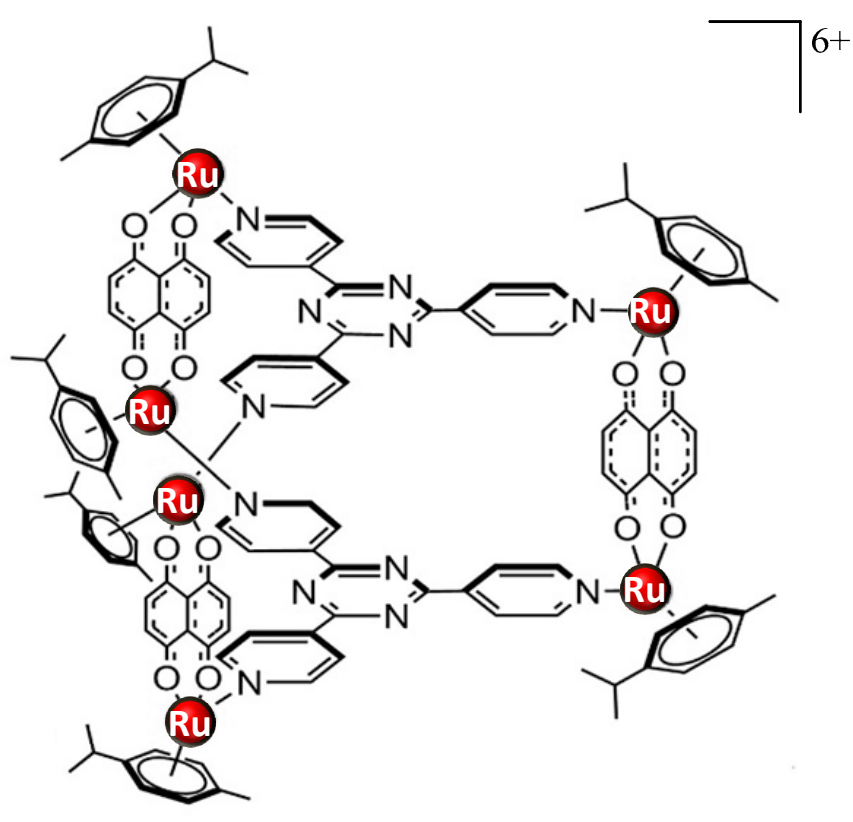

(a)

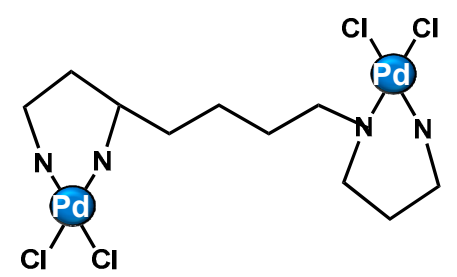

(b)<smiles>CC(=O)C(C(C)=O)C1(S(C)=O)OC(C)C(C)C(C)O1</smiles>

(c)

Figure 4. Structure of metal complexes different from cisplatin and studied through ${ }^{1} \mathrm{H}$ NMR metabolomic approaches. (a) Ruthenium metallaprism; (b) $\mathrm{Pd}_{2}$ spermine; (c) $\left[\mathrm{Pt}\left(O, O^{\prime}\right.\right.$-acac $)(\gamma$ acac)(DMS)], Ptac2S.

Other NMR-metabolomics studies have focused on Palladium (Pd) compounds. Iamego et al. describes metabolomic studies of a $\mathrm{Pd}_{2}$ Spermine $\left(\mathrm{Pd}_{2} \mathrm{Spm}\right)$ complex (Figure $\left.4 \mathrm{~b}\right)$ on osteosarcoma MG-63 and osteoblastic HOb cells. The aim of their work was to assess the impact of the potential palladium drug on cell metabolism in comparison with cisplatin. The authors have shown that despite its higher cytotoxicity, $\mathrm{Pd}_{2} \mathrm{Spm}$ induced a lower (and reversible) metabolic impact on MG-63 cells, as well as the absence of apoptosis; however, it induced significant deviations in the osteoblastic amino acid metabolism. Moreover, when administrated in combination with doxorubicin and methotrexate, the complex $\mathrm{Pd}_{2}$ Spm induced strong metabolic deviations in lipids, choline compounds, amino acids, nucleotides, and compounds related to anti-oxidative mechanisms (e.g., glutathione, inositol, and hypoxanthine). These results were similar to those observed for cisplatin treatments in combination with doxorubicin and methotrexate. These findings open promising perspectives related to the impact of $\mathrm{Pd}_{2} \mathrm{Spm}$ on osteosarcoma cellular metabolism, particularly in drug combination protocols. The lipid metabolism, glycosylation, and amino acid metabolisms have emerged as relevant features for targeted studies, to understand further the potential anticancer mechanism of combined $\mathrm{Pd}_{2} \mathrm{Spm}$ [67].

NMR metabolomics can be applied also in the study of drug treatment resistance. Recently, our group reported an ${ }^{1} \mathrm{H}-\mathrm{NMR}$-based metabolomic study to evaluate the response of a cisplatin-resistant epithelial ovarian carcinoma cell line, SKOV-3, to a new promising $\mathrm{Pt}(\mathrm{II}) \operatorname{drug},\left[\mathrm{Pt}\left(O, \mathrm{O}^{\prime}\right.\right.$-acac $)(\gamma-$ acac)(DMS)], or Ptac2S (Figure 4c). This complex is interesting for its higher anticancer activity, enhanced pharmacokinetics, bio-distribution, and tolerability showed in comparison to cisplatin. This is produced by a non-genomic mechanism of action, as previously demonstrated both in vitro and in vivo [77-82]. The observed higher cytotoxicity of Ptac2S with respect to cisplatin in the cisplatin-resistant SKOV-3 cells, evaluated by a preliminary MTT (3-(4,5-dimethylthiazol-2-yl)-2,5-diphenyltetrazolium bromide) 
assay, led us to investigate further the action mechanism of this complex, which is able to overcome cisplatin resistance.

Cell extracts (aqueous, as shown in Figure 5, and lipidic), as well as the recovered culture media for each condition (controls, Ptac2S, and cisplatin-treated) and time of treatment (6-24 h), were analysed by ${ }^{1} \mathrm{H}$ NMR. As expected, multivariate analysis of the NMR data gave new insights about the mechanism of action of Ptac2S. Through a careful investigation of the metabolites responsible for the observed differences between the two treatments (Ptac2S and cisplatin), it was found that the exposure of SKOV-3 cells to Ptac2S caused a cell membrane modification, a decrease of Krebs cycle efficiency, and an inhibition of the protein catabolism. By metabolomic analysis, the role of pyruvate was also found to be crucial in the antitumor activity of Ptac2S. The SKOV-3 cells' metabolic behaviour after the Ptac2S treatment suggests a possible "reversal of the Warburg effect" through the inhibition of lactate synthesis (probably due to a modulation of the lactate dehydrogenase activity). Furthermore, the different lipidic profile in $\mathrm{Ptac}_{2} \mathrm{~S}$, with respect to cisplatin-treated cells, indicates a possible cell death mechanism different from apoptosis (the known cisplatin-induced mechanism of death) [81]. In this context, biological assays have recently demonstrated that Ptac2S induces autophagy in another cisplatin-resistant cancer cell line (Caki-1, a renal cancer cell line) [82]. These findings parallel the results derived through the NMR metabolomic analysis, confirming the power of the technique in the investigation of mechanisms of action.
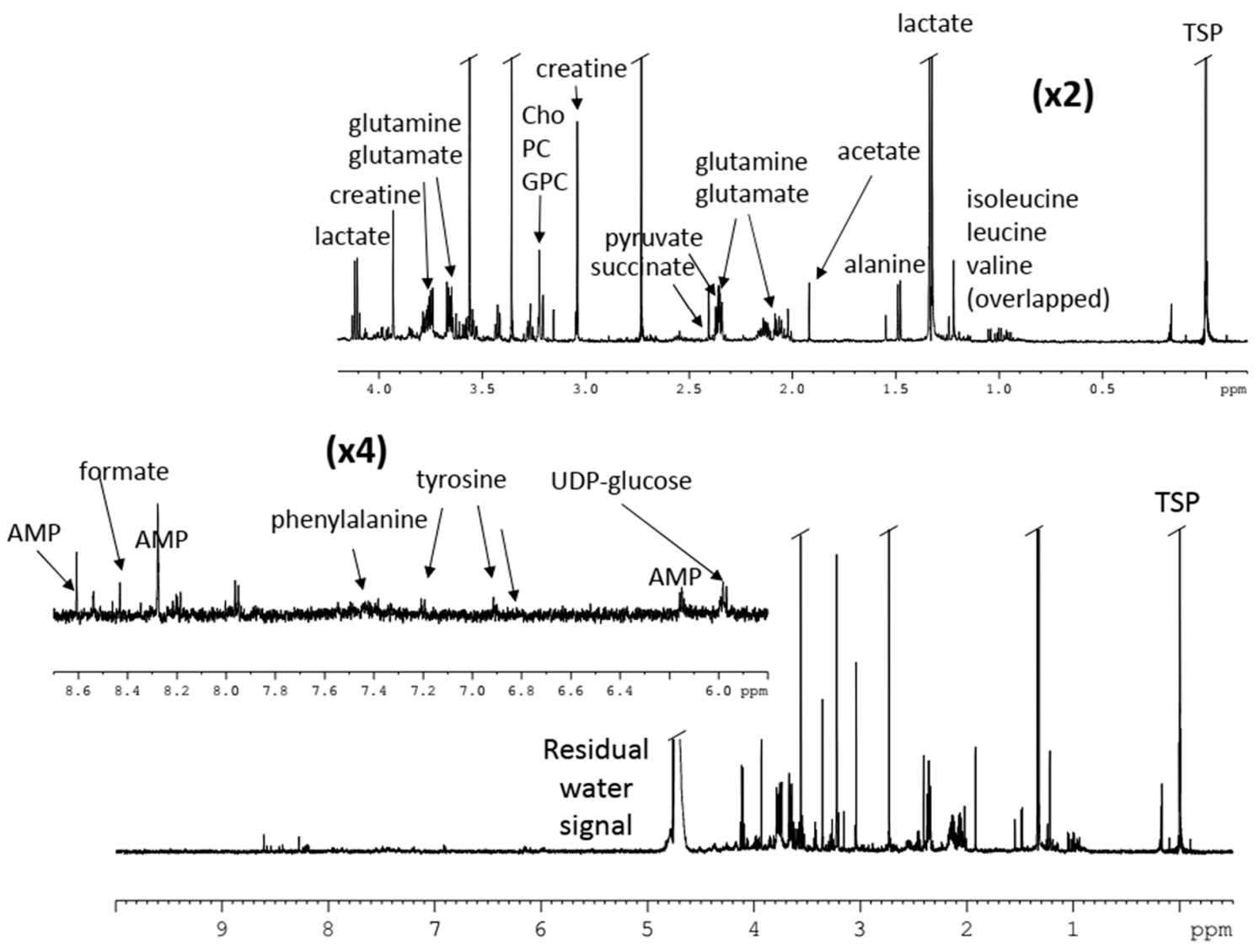

Figure 5. ${ }^{1} \mathrm{H}$ NMR spectrum of aqueous extracts of cisplatin-resistant, epithelial ovarian carcinoma cell line SKOV-3 cells treated with $\left[\mathrm{Pt}\left(O, \mathrm{O}^{\prime}\right.\right.$-acac $)(\gamma$-acac $\left.)(\mathrm{DMS})\right], \mathrm{Ptac} 2 \mathrm{~S}$ [81].

\section{Conclusions}

In this review, the great potential and application of NMR-based metabolomics in the metal-based drug research field are discussed [67,69-77,81]. Despite pitfalls due to the variability of cell cultures and the environmental influence, NMR metabolomics have excellent reproducibility and quantitative 
accuracy. This is made possible for standardized experimental methods (generally referred to controls), as well as for the stability of analytical platforms, both with the powerful statistical databases of metabolic data and efficient multivariate statistical methods that have also simplified the high complexity of the dataset. The use of NMR-based metabolomic approaches in the investigation of a metal drug action mechanism or for assessing tumour response to anticancer metal agents is a recent, fast-growing tool. The future perspectives are even more interesting. Due to the low work-up required, high data reproducibility, and high throughput, NMR spectroscopy is an optimal detection technique in metabolomics studies. Indeed, metal drug-induced side effects, treatment response prediction (thus also eventually resistance), and action mechanism key information for known and new compounds could be easily obtained using NMR-based metabolomics. A greater use of this technique could lead to personalized medicine, thus allowing high-throughput screening of pharmacologically active molecules, as well as lower doses chemotherapy. Furthermore, NMR-based metabolomics could also play an important role in clinical trials, preventing or reducing unwanted side effects of metal anticancer drugs by the early detection of metabolic dysfunctions in bio-fluids.

Author Contributions: F.D.C. and L.D.C. prepared the original draft; F.P.F. and M.B. wrote, reviewed, and edited; F.P.F. supervised and acquired funding. All authors read and approved the final manuscript.

Funding: This research received no external funding.

Acknowledgments: Consorzio Interuniversitario di Ricerca in Chimica dei Metalli nei Sistemi Biologici (CIRCMSB), Bari (Italy), and MEtabolic Profile-Assisted PREgnancy DIabetes Screening (MEPAPREDIS, POR PUGLIA FESR-FSE 2014-2020) are acknowledged for support.

Conflicts of Interest: The authors declare no conflict of interest.

\section{References}

1. Zwart, H. Human Genome Project: History and assessment. In International Encyclopedia of Social \& Behavioral Sciences, 2nd ed.; Elsevier: Oxford, UK, 2015; pp. 311-317.

2. Horgan, R.P.; Kenny, L.C. 'Omic'technologies: Genomics, transcriptomics, proteomics and metabolomics. Obstet. Gynaecol. 2011, 13, 189-195.

3. Deidda, M.; Piras, C.; Bassareo, P.P.; Cadeddu Dessalvi, C.; Mercuro, G. Metabolomics, a promising approach to translational research in cardiology. IJC Metab. Endocr. 2015, 9, 31-38. [CrossRef]

4. Beger, R.D. A review of applications of metabolomics in cancer. Metabolites 2013, 3, 552-574. [CrossRef] [PubMed]

5. Nicholson, J.K.; Lindon, J.C.; Holmes, E. Metabonomics': Understanding the metabolic responses of living systems to pathophysiological stimuli via multivariate statistical analysis of biological NMR spectroscopic data. Xenobiotica 1999, 29, 1181-1189. [CrossRef] [PubMed]

6. Oliver, S.G. Functional genomics: Lessons from yeast. Philos. Trans. R. Soc. Lond. B Biol. Sci. 2002, 357, 17-23. [CrossRef] [PubMed]

7. Everett, J.R. Drug Discovery and Development: The Role of NMR. eMag. Res. 2015, 4, 137-149.

8. Lindon, J.; Nicholson, J.; Holmes, E.; Everett, J.R. Metabonomics: Metabolic processes studied by NMR spectroscopy of biofluids. Concepts Magn. Reson. 2000, 12, 289-320. [CrossRef]

9. Fiehn, O. Metabolomics-the link between genotypes and phenotypes. Plant Mol. Biol. 2002, 48, 155-171. [CrossRef]

10. Lindon, J.C.; Nicholson, J.K.; Holmes, E. Metabonomics and Metabolomics Techniques and Their Applications in Mammalian Systems. In The Handbook of Metabonomics and Metabolomics, 1st ed.; Elsevier Science: Amsterdam, The Netherlands, 2006; pp. 1-33.

11. Jones, O.A.H.; Lee, D.R.; Maguire, M.L. Sample Preparation Techniques in Metabolomics and Drug Discovery. In Sample Preparation in Biological Mass Spectrometry; Ivanov, A.R., Lazarev, A.V., Eds.; Springer: Dordrecht, The Netherlands; Heidelberg, Germany; London, UK; New York, NY, USA, 2011; part XIII; pp. 853-910.

12. Nicholson, J.K.; Lindon, J.C. Systems biology: Metabonomics. Nature 2008, 455, 1054-1056. [CrossRef]

13. Bray, F.; Ferlay, J.; Soerjomataram, I.; Siegel, R.L.; Torre, L.A.; Jemal, A. Global cancer statistics 2018: GLOBOCAN estimates of incidence and mortality worldwide for 36 cancers in 185 countries. CA Cancer J. Clin. 2018, 68, 394-424. [CrossRef] [PubMed] 
14. Potter, M.; Newport, E.; Morten, K.J. The Warburg effect: 80 years on. Biochem. Soc. Trans. 2016, 44, $1499-1505$. [CrossRef] [PubMed]

15. Warburg, O.; Wind, F.; Negelein, E. The metabolism of tumors in the body. J. Gen. Physiol. 1927, 8, 519-530. [CrossRef] [PubMed]

16. Cori, C.A.; Cori, G.T. The carbohydrate metabolism of tumours. J. Biol. Chem. 1925, 65, 397-405.

17. Migliore, L.; Coppedè, F. Genetic and environmental factors in cancer and neurodegenerative diseases. Mutat. Res. 2002, 512, 135-153. [CrossRef]

18. Ladley, S.E.; Hales, D.B. Ovarian Cancer Cell Survival and Proliferation Is Mediated by the Warburg Effect. Biol. Reprod. 2011, 85, 197. [CrossRef]

19. Fu, Y.; Liu, S.; Yin, S.; Niu, W.; Xiong, W.; Tan, M.; Li, G.; Zhou, M. The reverse Warburg effect is likely to be an Achilles' heel of cancer that can be exploited for cancer therapy. Oncotarget 2017, 8, 57813-57825. [CrossRef] [PubMed]

20. Doherty, J.R.; Cleveland, J.L. Targeting lactate metabolism for cancer therapeutics. J. Clin. Investig. 2013, 123, 3685-3692. [CrossRef] [PubMed]

21. Hamanaka, R.B.; Chandel, N.S. Targeting glucose metabolism for cancer therapy. J. Exp. Med. 2012, 209, 211-215. [CrossRef] [PubMed]

22. Pelicano, H.; Martin, D.S.; Xu, R.-H.; Huang, P. Glycolysis inhibition for anticancer treatment. Oncogene 2006, 25, 4633-4646. [CrossRef]

23. Zhao, D.; Zou, S.W.; Liu, Y.; Zhou, X.; Mo, Y.; Wang, P.; Xu, Y.H.; Dong, B.; Xiong, Y.; Lei, Q.Y.; et al. Lysine-5 acetylation negatively regulates lactate dehydrogenase $\mathrm{A}$ and is decreased in pancreatic cancer. Cancer Cell 2013, 23, 464-476. [CrossRef]

24. Luengo, A.; Gui, D.Y.; Vander Heiden, M.G. Targeting Metabolism for Cancer Therapy. Cell. Chem. Biol. 2017, 24, 1161-1180. [CrossRef] [PubMed]

25. Hay, N. Reprogramming glucose metabolism in cancer: Can it be exploited for cancer therapy? Nat. Rev. Cancer 2016, 16, 635-649. [CrossRef] [PubMed]

26. Shim, H.; Dolde, C.; Lewis, B.C.; Wu, C.S.; Dang, G.; Jungmann, R.A.; Dalla-Favera, R.; Dang, C.V. c-Myc transactivation of LDH-A: Implications for tumor metabolism and growth. Proc. Natl. Acad. Sci. USA 1997, 94, 6658-6663. [CrossRef] [PubMed]

27. Zhao, Y.; Butler, E.B.; Tan, M. Targeting cellular metabolism to improve cancer therapeutics. Cell Death Dis. 2013, 4, e532. [CrossRef] [PubMed]

28. Sakashita, M.; Aoyama, N.; Minami, R.; Maekawa, S.; Kuroda, K.; Shirasaka, D.; Ichihara, T.; Kuroda, Y.; Maeda, S.; Kasuga, M. Glut1 expression in T1 and T2 stage colorectal carcinomas: Its relationship to clinicopathological features. Eur. J. Cancer. 2001, 37, 204-209. [CrossRef]

29. Grover-McKay, M.; Walsh, S.A.; Seftor, E.A.; Thomas, P.A.; Hendrix, M.J.C. Role for glucose transporter 1 protein in human breast cancer. Pathol. Oncol. Res. 1998, 4, 115-120. [CrossRef] [PubMed]

30. Wu, M.; Neilson, A.; Swift, A.L.; Moran, R.; Tamagnine, J.; Parslow, D.; Armistead, S.; Lemire, K.; Orrell, J.; Teich, J.; et al. Multiparameter metabolic analysis reveals a close link between attenuated mitochondrial bioenergetic function and enhanced glycolysis dependency in human tumor cells. Am. J. Physiol. Cell Physiol. 2007, 292, C125-C136. [CrossRef] [PubMed]

31. Lai, J.-H.; Jan, H.-J.; Liu, L.-W.; Lee, C.-C.; Wang, S.-G.; Hueng, D.-Y.; Cheng, Y.Y.; Lee, H.M.; Ma, H.I. Nodal regulates energy metabolism in glioma cells by inducing expression of hypoxia-inducible factor $1 \alpha$. Neuro-Oncol. 2013, 15, 1330-1341. [CrossRef] [PubMed]

32. Michelakis, E.D.; Sutendra, G.; Dromparis, P.; Webster, L.; Haromy, A.; Niven, E.; Maguire, C.; Gammer, T.L.; Mackey, J.R.; Fulton, D.; et al. Metabolic modulation of glioblastoma with dichloroacetate. Sci. Transl. Med. 2010, 2, 31ra34. [CrossRef] [PubMed]

33. Vander Heiden, M.G.; Cantley, L.C.; Thompson, C.B. Understanding the Warburg effect: The metabolic requirements of cell proliferation. Science 2009, 324, 1029-1033. [CrossRef]

34. Griffin, J.L.; Shockcor, J.P. Metabolic profiles of cancer cells. Nat. Rev. Cancer 2004, 4, 551-561. [CrossRef] [PubMed]

35. Struck, W.; Waszczuk-Jankowska, M.; Kaliszan, R.; Markuszewski, M.J. The state-of-the-art determination of urinary nucleosides using chromatographic techniques "Hyphenated" with advanced bioinformatics methods. Anal. Bioanal. Chem. 2011, 410, 2039-2050. [CrossRef] [PubMed] 
36. Zambonin, C.G.; Aresta, A.; Palmisano, F.; Specchia, G.; Liso, V. Liquid chromatography determination of urinary 5-methyl-2'-deoxycytidine and psuedouridine as potential biomarkers for leukaemia. J. Pharm. Biomed. Anal. 1999, 21, 1045-1051. [CrossRef]

37. Sasco, A.J.; Rey, F.; Reynaud, C.; Bobin, Y.L.; Clavel, M.; Niveleau, A. Breast cancer prognostic significance of some modified urinary nucleosides. Cancer Lett. 1996, 108, 157-162. [CrossRef]

38. Zheng, Y.F.; Kong, H.W.; Xiong, J.H.; Lv, S.; Xu, G.W. Clinical significance and prognostic value of urinary nucleosides in breast cancer patients. Clin. Biochem. 2005, 38, 24-30. [CrossRef] [PubMed]

39. Woo, H.M.; Kim, K.M.; Choi, M.H.; Jung, B.H.; Lee, J.; Kong, G.; Nam, S.J.; Kim, S.; Bai, S.W.; Chung, B.C. Mass spectrometry based metabolomic approaches in urinary biomarker study of women's cancers. Clin. Chem. Acta 2009, 400, 63-69. [CrossRef]

40. Zheng, Y.F.; Yang, J.; Zhao, X.J.; Feng, B.; Kong, H.W.; Chen, Y.J.; Lv, S.; Zheng, M.H.; Xu, G.W. Urinary nucleosides as biological markers for patients with colorectal cancer. World J. Gastroenterol. 2005, 11, 3871-3876. [CrossRef]

41. Yang, J.; Xu, G.; Zheng, Y.; Kong, H.; Pang, T.; Lv, S.; Yang, Q. Diagnosis of liver cancer using hplc-based metabonomics avoiding false-positive result from hepatitis and hepatocirrhosis diseases. J. Chrom. B. 2004, 813, 59-65. [CrossRef]

42. Kobayashi, T.; Nishiumi, S.; Ikeda, A.; Yoshie, T.; Sakai, A.; Matsubara, A.; Izumi, Y.; Tsumura, H.; Tsuda, M.; Nishisaki, H.; et al. A novel serum metabolomics-based diagnostic approach to pancreatic cancer. Cancer Epidemiol. Biomark. Prev. 2013, 22, 571-579. [CrossRef]

43. Ikeda, A.; Nishiumi, S.; Shinohara, M.; Yoshie, T.; Hatano, N.; Okuno, T.; Bamba, T.; Fukusaki, E.; Takenawa, T.; Azuma, T.; et al. Serum metabolomics as a novel diagnostic approach for gastrointestinal cancer. Biomed. Chromatogr. 2012, 26, 548-558. [CrossRef]

44. Odunsi, K.; Wollman, R.M.; Ambrosone, C.B.; Hutson, A.; McCann, S.E.; Tammela, J.; Geisler, J.P.; Miller, G.; Sellers, T.; Cliby, W.; et al. Detection of epithelial ovarian cancer using ${ }^{1} \mathrm{H}-\mathrm{NMR}$-based metabonomics. Int. J. Cancer 2005, 113, 782-788. [CrossRef] [PubMed]

45. Osl, M.; Drreiseitl, S.; Pfeifer, B.; Weinberger, K.; Klocker, H.; Bartsch, G.; Schafer, G.; Tilg, B.; Graber, A. A new rule-based algorithm for identifying metabolic markers in prostate cancer using tandem mass spectrometry. Bioinformatics 2008, 24, 2908-2914. [CrossRef] [PubMed]

46. Gao, P. C-myc suppression of mir-23a/b enhances mitochondrial glutaminase expression and glutamine metabolism. Nature 2009, 458, 762-765. [CrossRef] [PubMed]

47. Wang, J.; Yu, L.F.; Shen, P.; Wang, S.F. Analysis of serum metabolome of patients with breast cancer by gas chromatography-mass spectrometry. Zhejiang Da Xue Bao Yi Xue Ban 2009, 38, 478-484.

48. Beger, R.; Schnackenberg, L.; Holland, R.; Li, D.; Dragan, Y. Metabonomic models of human pancreatic cancer using 1D proton NMR spectra of lipids in plasma. Metabolomics 2006, 2, 125-134. [CrossRef]

49. Yan, S.K.; Wei, B.J.; Lin, Z.Y.; Yang, Y.; Zhou, Z.T.; Zhang, W.D. A metabonomic approach to the diagnosis of oral squamous cell carcinoma, oral clichen planus and oral leukoplakia. Oral Oncol. 2008, 44, 477-483. [CrossRef] [PubMed]

50. Kim, R.; Coates, J.; Bowles, T.; McNerney, G.; Sutcliffe, J.; Jung, I.; Gandour-Edwaeds, R.; Chuang, F.; Bold, R.; Kung, H. Arginine deiminase as a novel therapy for prostate cancer induces autophagy and caspase-independent apoptosis. Cancer Res. 2009, 69, 700-708. [CrossRef]

51. Wen, S.; Zhan, B.; Feng, J.; Hu, W.; Lin, X.; Bai, J.; Huang, H. Non-invasively predicting differentiation of pancreatic cancer through comparative serum metabonomic profiling. BMC Cancer 2017, 17, 708/1-708/11. [CrossRef]

52. Zacharias, H.; Altenbuchinger, M.; Gronwald, W. Statistical analysis of NMR metabolic fingerprints: established methods and recent advances. Metabolites 2018, 8, 47. [CrossRef]

53. Eriksson, L.; Byrne, T.; Johansson, E.; Trygg, J.; Vikström, C. Multi-and Megavariate Data Analysis Basic Principles and Applications, 3rd ed.; Umetrics Academy: Malmö, Sweden, 2013.

54. Eastment, H.; Krzanowski, W. Cross-validatory choice of the number of components from a principal component analysis. Technometrics 1982, 24, 73-77. [CrossRef]

55. Bro, R.; Smilde, A.K. Principal component analysis. Anal. Methods 2014, 6, 2812-2831. [CrossRef]

56. Trygg, J.; Wold, S. Orthogonal projections to latent structures (O-PLS). J. Chemom. 2002, 16, 119-128. [CrossRef]

57. Mechanism matters, Editorial. Nat. Med. 2010, 16, 347. [CrossRef] [PubMed] 
58. Doody, R.S.; Gavrilova, S.I.; Sano, M.; Thomas, R.G.; Aisen, P.S.; Bachurin, S.O.; Seely, L.; Hung, D. Effect of dimebon on cognition, activities of daily living, behaviour, and global function in patients with mild-to-moderate Alzheimer's disease: A randomised, double-blind, placebo-controlled study. Lancet 2008, 372, 207-215. [CrossRef]

59. Rattner, J.; Bathe, O.F. Monitoring for Response to Antineoplastic Drugs: The Potential of a Metabolomic Approach. Metabolites 2017, 7, 60. [CrossRef] [PubMed]

60. Rosenberg, B.; Van Camp, L. The successful regression of large solid sarcoma 180 tumors by platinum compounds. Cancer Res. 1970, 30, 1799-1802. [PubMed]

61. Lovejoy, K.S.; Lippard, S.J. Non-traditional platinum compounds for improved accumulation, oral bioavailability, and tumor targeting. Dalton Trans. 2009, 48, 10651-10659. [CrossRef] [PubMed]

62. Kostova, I. Ruthenium complexes as anticancer agents. Curr. Med. Chem. 2006, 13, 1085-1107. [CrossRef] [PubMed]

63. Braga, S.S.; Marques, J.; Heister, E.; Diogo, C.V.; Oliveira, P.J.; Paz, F.A.A.; Santos, T.M.; Marques, M.P.M. Carriers for metal complexes on tumour cells: The effect of cyclodextrins vs CNTs on the model guest phenanthroline-5,6-dione trithiacyclononane ruthenium- (II) chloride. BioMetals 2014, 27, 507-525. [CrossRef]

64. Kostova, I. Titanium and vanadium complexes as anticancer agents. Anti-Cancer Agents Med. Chem. 2009, 9, 827-842. [CrossRef]

65. Messori, L.; Scaletti, F.; Massai, L.; Cinellu, M.A.; Gabbiani, C.; Vergara, A.; Merlino, A. The mode of action of anticancer gold-based drugs: A structural perspective. Chem. Commun. 2013, 49, 10100-10102. [CrossRef] [PubMed]

66. Ray, S.; Mohan, R.; Singh, J.K.; Samantaray, M.K.; Shaikh, M.M.; Panda, D.; Ghosh, P. Anticancer and antimicrobial metallopharmaceutical agents based on palladium, gold, and silver Nheterocyclic carbene complexes. J. Am. Chem. Soc. 2007, 129, 15042-15053. [CrossRef] [PubMed]

67. Lamego, I.; Marques, M.P.M.; Duarte, I.F.; Martins, A.S.; Oliveira, H.; Gil, A.M. Impact of the $\mathrm{Pd}_{2} \mathrm{Spermine}$ Chelate on Osteosarcoma Metabolism: An NMR Metabolomics Study. J. Proteome Res. 2017, 16, 1773-1783. [CrossRef] [PubMed]

68. Marques, M.P.M. Platinum and palladium polyamine complexes as anticancer agents: The structural factor. ISRN Spectrosc. 2013, 2013, 1-29. [CrossRef]

69. Portilla, D.; Li, S.; Nagothu, K.K.; Megyesi, J.; Kaissling, B.; Schnackenberg, L.; Safirstein, R.L.; Beger, R.D. Metabolomic study of cisplatin-induced nephrotoxicity. Kidney Int. 2006, 69, 2194-2204. [CrossRef] [PubMed]

70. Wen, H.; Yang, H.-J.; Choi, M.-J.; Kwon, H.N.; Kim, M.A. Soon-Sun Hong and Sunghyouk Park. Identification of Urinary Biomarkers Related to Cisplatin- Induced Acute Renal Toxicity Using NMR-Based Metabolomics. Biomol. Ther. 2011, 19, 38-44. [CrossRef]

71. Ferrier, J.; Bayet-Robert, M.; Pereira, B.; Balayssac, D. A Polyamine-Deficient Diet Prevents Oxaliplatin-Induced Acute Cold and Mechanical Hypersensitivity in Rats. PLoS ONE 2013, 8, e77828. [CrossRef]

72. Jiang, L.; Lee, S.C.; Ng, T.C. Pharmacometabonomics Analysis Reveals Serum Formate and Acetate Potentially Associated with Varying Response to Gemcitabine-Carboplatin Chemotherapy in Metastatic Breast Cancer Patients. J. Proteome Res. 2018, 17, 1248-1257. [CrossRef]

73. Xu, S.; Zhou, Y.; Geng, H.; Song, D.; Tang, J.; Zhu, X.; Yu, D.; Hu, S.; Cui, Y. Serum Metabolic Profile Alteration Reveals Response to Platinum-Based Combination Chemotherapy for Lung Cancer: Sensitive Patients Distinguished from Insensitive ones. Sci. Rep. 2017, 7, 17524. [CrossRef]

74. Duarte, I.F.; Ladeirinha, A.F.; Lamego, I.; Gil, A.M.; Carvalho, L.; Carreira, I.M.; Melo, J.B. Potential Markers of Cisplatin Treatment Response Unveiled by NMR Metabolomics of Human Lung Cells. Mol. Pharm. 2013, 10, 4242-4251. [CrossRef]

75. Lamego, I.; Duarte, I.F.; Marques, M.P.M.; Gil, A.M. Metabolic Markers of MG-63 Osteosarcoma Cell Line Response to Doxorubicin and Methotrexate Treatment: Comparison to Cisplatin. J. Proteome Res. 2014, 13, 6033-6045. [CrossRef] [PubMed]

76. Liu, S.; Wang, W.; Zhou, X.; Gu, R.; Ding, Z. Dose responsive effects of cisplatin in L02 cells using NMR-based metabolomics. Environ. Toxicol. Pharmacol. 2014, 37, 150-157. [CrossRef] [PubMed]

77. Vermathen, M.; Paul, L.E.H.; Diserens, G.; Vermathen, P.; Furrer, J. ${ }^{1}$ H HR-MAS NMR based metabolic profiling of cells in response to treatment with a hexacationic ruthenium metallaprism as potential anticancer drug. PLoS ONE 2015, 10, e0128478. [CrossRef] [PubMed] 
78. De Pascali, S.A.; Papadia, P.; Ciccarese, A.; Pacifico, C.; Fanizzi, F.P. First examples of diketonate platinum II complexes with sulfoxide ligands. Eur. J. Inorg. Chem. 2005, 5, 788-796. [CrossRef]

79. Muscella, A.; Calabriso, N.; De Pascali, S.A.; Urso, L.; Ciccarese, A.; Fanizzi, F.P.; Migoni, D.; Marsigliante, S. New platinum (II) complexes containing both an $\mathrm{O}, \mathrm{O}$-chelated acetylacetonate ligand and a sulfur ligand in the platinum coordination sphere induce apoptosis in HeLa cervical carcinoma cells. Biochem. Pharmacol. 2007, 74, 28-40. [CrossRef]

80. De Pascali, S.A.; Lugoli, F.; De Donno, A.; Fanizzi, F.P. Mutagenic tests confirm that new acetylacetonate $\mathrm{Pt}(\mathrm{II})$ complexes induce apoptosis in cancer cells interacting with non genomic biological targets. Metal-Based Drugs 2011, 2011, 763436. [CrossRef]

81. De Castro, F.; Benedetti, M.; Antonaci, G.; Del Coco, L.; De Pascali, S.A.; Muscella, A.; Marsigliante, S.; Fanizzi, F.P. Response of cisplatin resistant SKOV-3 cells to $[\mathrm{Pt}(\mathrm{O}, \mathrm{O}$-acac $)(\gamma$-acac $)(\mathrm{DMS})]$ Treatment revealed by a metabolomic ${ }^{1} \mathrm{H}-\mathrm{NMR}$ study. Molecules 2018, 23, 2301. [CrossRef] [PubMed]

82. Antonaci, G.; Cossa, L.G.; Muscella, A.; Vetrugno, C.; De Pascali, S.A.; Fanizzi, F.P.; Marsigliante, S. $\left[\mathrm{Pt}\left(O, \mathrm{O}^{\prime}\right.\right.$-acac $)(\gamma$-acac $\left.)(\mathrm{DMS})\right]$ induces autophagy in Caki-1 renal cancer cells. Biomolecules 2019, 9, 92. [CrossRef] [PubMed]

(C) 2019 by the authors. Licensee MDPI, Basel, Switzerland. This article is an open access article distributed under the terms and conditions of the Creative Commons Attribution (CC BY) license (http://creativecommons.org/licenses/by/4.0/). 